\title{
Orthodontic retention - three methods trialed
}

\section{Abstracted from \\ Edman Tynelius G, Petrén S, Bondemark L, Lilja-Karlander E.}

Five-year postretention outcomes of three retention methods - a randomized controlled trial. Eur J Orthod 2015; 37: 345-353.

Address for correspondence: Gudrun Edman Tynelius, Harvigslund Stubbarp 112,

S-274 91 Skurup, Sweden. E-mail: gudrunedmantynelius@gmail.com

\section{Question: What is the effect of three retention strategies on orthodontic relapse?}

Design Single centre randomised controlled trial.

Intervention Patients treated by a single orthodontist were randomised to one of three retention methods: removable vacuumformed retainer (VFR) covering the palate and the maxillary anterior teeth from canine-to-canine and bonded canine-to-canine retainer in the lower arch (group V-CTC); maxillary VFR combined with stripping of the lower anterior teeth (group V-S); prefabricated positioner covering all erupted teeth in the maxilla and the mandible (group P). All retention appliances were provided within one hour of debonding. Outcome measure Dental study casts were taken before treatment, at appliance removal and five years or more out of retention. Little's irregularity index, intercanine and intermolar width, arch length and overbite/overjet were recorded.

Results Twenty-five patients were randomised to each group with 69 completing the two-year retention period (24 in V-CTC group; 23 in $\mathrm{V}-\mathrm{S}$ group; 22 in $\mathrm{P}$ group). Forty-nine patients were available five years post retention (16 in V-CTC group; 17 in V-S group; 16 in P group). No significant differences were found between the groups.

Conclusions After five years or more out of retention, the three retention methods had achieved equally favourable clinical results. Thus a maxillary VFR combined with a bonded canine-to-canine retainer in the mandible, a maxillary VFR combined with stripping of the mandibular anterior teeth and a prefabricated positioner can all be recommended.

\section{Commentary}

Following a course of orthodontic treatment some relapse of tooth position is common ${ }^{1}$ and a period of retention is required in order to ensure long-term stability. Although the degree of change is highest during the first ten years post orthodontic treatment, relapse in the anterior mandible has been demonstrated to be ongoing between ten and 20 years post retention phase. ${ }^{2}$ Therefore long term retention is often required, ${ }^{3}$ with many now advocating life long retention.

This year a systematic review by The Cochrane Collaboration concluded 'there is insufficient high quality evidence to make recommendations on retention procedures for stabilising tooth position after treatment with orthodontic braces. Further high quality RCTs are needed. ${ }^{4}$

The aim of this randomised controlled trial was to add to the sparse evidence base and evaluate and compare the effects of three different retention strategies on inhibiting relapse at least five years post retention.

Assessing tooth position five years post retention acknowledges that active and significant change continues for years, even decades, after orthodontic therapy. ${ }^{2}$ As with all long-term studies the problem of loss to follow up is high, with $35 \%$ of patients failing to complete the trial.

However, the retention methods chosen for inclusion in the study are unorthodox in UK practice, where a vacuum-formed retainer (VFR) covering all occlusal surfaces or a maxillary bonded retainer maybe more frequently used. The use of a positioner for orthodontic retention is rare in UK practice. This is a single centre study, performed in Sweden, which will be of limited benefit to many UK practitioners wishing to compare their preferred retention method to alternatives. It would be beneficial to replicate this randomised control trial in a multi-centre setting in the UK, comparing retention methods more commonly used in UK orthodontic practice.

The study found that after five years or more out of retention, each method had achieved equally favourable clinical results, and thus, the authors conclude that all three methods can be recommended. However, the results showed that small changes of tooth position have occurred, with an average maxillary Little's index of $1.8-2.6 \mathrm{~mm}$ and mandibular Little's index of $2.0-3.4 \mathrm{~mm}$. Were the patients happy with this small, but measurable, amount of relapse? If patient satisfaction regarding tooth position at five years was low, the opposite could be argued; that none of the above retention methods can be recommended, ie one could conclude that all methods were as unsuccessful as each other. 
Additional considerations must be taken into account when selecting a retention method, such as whether to adopt a removable or fixed retainer, with the latter having the advantage of minimal need for patient compliance. ${ }^{5}$ The paper acknowledges that the success of removable retention methods depends largely on patient compliance. Removable appliances may demonstrate higher levels of success when their provision is properly considered and tailored to the patient compliance, rather than randomly allocated, as in this trial.

The paper makes no mention of patient satisfaction levels regarding comfort or ease of wear of each retention method. It also does not record the frequency of failure of the bonded wire retainers, which have been reported as between 12 to $50 \%{ }^{5}$, or loss of the removable appliances, which can add to clinical time and additional financial considerations.

This study can be credited as the first randomised controlled trial to find that stripping of mandibular anterior teeth alone provides equivalent retention to bonded appliances. This method requires no fixed or removable retention, thus reducing chair time, cost and the issue of lost appliances.

As the first randomised controlled trial to compare the success of multiple retention methods in the long-term, this study has great potential to provide an evidence base for the selection of the best treatment protocol, which remains a subjective issue. ${ }^{6}$ It is indeed a well-designed study, with a clearly calculated sample size and a well-written paper.

J Faith Cope ${ }^{a}$ and Thomas Lamont ${ }^{b}$ ${ }^{a}$ Glasgow Dental Hospital, Glasgow, Scotland

${ }^{b}$ Dundee Dental School, Dundee, Scotland

1. Horowitz SL, Hixon EH. Physiologic recovery following orthodontic treatment. Am J Orthod 1969; 55: 1-4.

2. Little RM, Riedel RA, Artun J. An evaluation of changes in mandibular anterior alignment from 10 to 20 years postretention. Am / Orthod Dentofacial Orthop 1988; 93: 423-428.

3 Bolla E, Cozzani M, Doldo T, Fontana M. Failure evaluation after a 6-year retention period: a comparison between glass fiber-reinforced (GFR) and multistranded bonded retainers. Int Orthod 2012: 10: 16-28.

4. Littlewood SJ, Millett DT, Doubleday B, Bearn DR, Worthington HV. Retention procedures for stabilising tooth position after treatment with orthodontic braces. Cochrane Database Syst Rev 2016; 1: CD002283. doi: 10.1002/14651858. CD002283.pub4.

5. Iliadi A Kloukos D, Gkantidis N, Katsaros C, Pandis N. Failure of fixed orthodontic retainers: A systematic review. J Dent 2015; 43: 876-896.

6. Littlewood SJ, Millett DT, Doubleday B, Bearn DR, Worthington HV. Retention procedures for stabilising tooth position after treatment with orthodontic braces. Cochrane Database Syst Rev 2006; 1: CD002283.

Evidence-Based Dentistry (2016) 17, 29-30. doi:10.1038/sj.ebd.6401157 